\title{
Humanistic Care Nursing Mode for Severe Hyperbilirubinemia Patients Who Undergoing Double Plasma Molecular Adsorption System and Plasma Exchange
}

\author{
Rou Wen ${ }^{1}$, Zhaolin Chen ${ }^{2,}$, Yanyun Chen ${ }^{1}$ \\ ${ }^{1}$ Artificial Liver Blood Purification Department, The First Affiliated Hospital of Jinan University, Guangzhou, China \\ ${ }^{2}$ Digestive Department, The First Affiliated Hospital of Jinan University, Guangzhou, China \\ Email address: \\ 729441864@qq.com (Rou Wen), 2009gznu@163.com (Zhaolin Chen), 499324016@qq.com (Yanyun Chen) \\ ${ }^{*}$ Corresponding author
}

To cite this article:

Rou Wen, Zhaolin Chen, Yanyun Chen. Humanistic Care Nursing Mode for Severe Hyperbilirubinemia Patients Who Undergoing Double Plasma Molecular Adsorption System and Plasma Exchange. American Journal of Nursing Science. Vol. 9, No. 3, 2020 , pp. $116-119$. doi: 10.11648/j.ajns.20200903.16

Received: March 26, 2020; Accepted: April 14, 2020; Published: April 23, 2020

\begin{abstract}
Objective: To assess humanistic care nursing mode for severe hyperbilirubinemia patients who undergoing double plasma molecular adsorption system and plasma exchange. Methods: 124 participants were diagnosed with severe hyperbilirubinemia and were received combined treatment, include that double plasma molecular adsorption system and Plasma exchange. They were randomly assigned to control group $(n=62)$ and intervention group $(n=62)$. We collect the participant information by Self-Rating Anxiety Scale, Self-rating depression scale and the Short Form-36. The information include the satisfaction of patients, anxiety status, depression status and quality of life. Result: In patient satisfaction research, the intervention group has more very good level assessment than that of control group (41 vs 32). Also, control group has more good level assessment than that of intervention group (18 vs 8$)$. In anxiety and depression research, intervention group has better improvement in anxiety status, it is from $56.4 \pm 6.7$ to $45.3 \pm 5.1$. In quality of life research, the intervention group has greater improvement than that of control group in every domain. Conclusion: humanistic care nursing mode provide better improvement in mental health aspect of patient. It significantly improved anxiety and depression, and improved overall quality of life after treatment.
\end{abstract}

Keywords: Humanistic Care Nursing, Hyperbilirubinemia, Double Plasma Molecular Adsorption System, Plasma Exchange

\section{Introduction}

The incidence of hyperbilirubinemia is high clinically, which is in some cases difficult to cure by medication, surgery or interventional therapies [1]. How to reduce the high concentration of bilirubin in the blood of patients has always been a focus of study for many researchers. Elevation of direct bilirubin levels may result from hepatobiliary dysfunction and be indicative of serious illness or pathology [2]. Diagnostic criteria for severe hyperbilirubinemia vary, with direct bilirubin levels that is $>2 \mathrm{mg} / \mathrm{dL}$ or accounts for greater than $20 \%$ of the total bilirubin after 2 weeks of life commonly cited $[3,4]$. Base on several reports, more accurate prediction of severe hyperbilirubinemia by combining the predischarge bilirubin level with clinical risk factors assessment than the use of either method alone and using various methods such as the prediction model or graphic tools $[5,6]$.

In the process of plasma exchange, plasma is separated and discarded, while cells with appropriate replacement solutions are reinfused back to the patient. Plasma exchange not only removes bilirubin, endotoxin and complement activators, but also replenishes albumin, coagulation factors and hepatic regenerative stimulating substance, which can correct metabolic disorder [7]. Base on recent report, plasma exchange has been regarded as the standard escalation therapy, based on one randomised, sham-controlled study in 22 
patients which showed a response rate of $42.1 \%$ [8]. In terms of the disadvantages of plasma exchange, the plasma exchange procedure is unspecific, and the loss of coagulation factors and other plasma constituents may cause complications, including thrombosis, bleeding, hypotension, and sepsis [9]. The double plasma molecular adsorption system is a new adsorption therapy in hyperbilirubinemia treatment. Base on traditional bilirubin adsorption therapy, the double plasma molecular adsorption system has an additional broad-spectrum adsorption column, it include neutral macroporous adsorption resin and ion exchange resin. Its functions are not only can adsorb medium-molecular toxins, but also absorb macro-molecular toxins in treatment process [10].

\section{Methods}

\subsection{Participants Enrollment and Study Methods}

All participants $(n=124)$ who were diagnosed with severe hyperbilirubinemia and were received combined treatment, such as double plasma molecular adsorption system and Plasma exchange. Their diagnosis of time is from February 2016 to January 2020. During this period, more patients were willing to join our study, but some patients did not meet our screening criteria, so they did not join our study. In the filter results, we have 124 participants, they meet our screening criteria. In study beginning, we randomly assign participants to two groups, that included a control group $(\mathrm{n}=62)$ and a intervention group $(n=62)$. In nursing service process, the participants of different groups have different nursing services in treatment period so that we can obtain the different data to contrast between two different nursing services. In control group, the participants receive general mode of care, the general mode of care is a model of care that most hospitals use for a long time. In intervention group, we use the humanistic care nursing mode to the participants, the humanistic care nursing mode provide more psychology-related nursing services to patients, such as mental health assessment, communication before treatment and follow-up after treatment. Additionally, we use interview and questionnaires to collect the information from participants, the information include the satisfaction of patients, anxiety status, depression status and quality of life. The collected data from the following questionnaires: Self-Rating Anxiety Scale (SAS), Self-rating depression scale (SDS) and the Short Form-36 (SF-36) [11-13].

Their inclusion criteria were: (1) Patient successfully completed the entire treatment process; (2) Patients volunteered to participate in follow-up; (3) Patient has no other serious illness. Their withdraw criteria were: (1) Patient's mental state is very poor; (2) Patients apply to withdraw from our study during treatment or nursing care.

\subsection{Statistical Analysis}

Our data analyzer performed the statistical analysis by SPSS 22.0. The $\mathrm{P}$ value, t-test and chi-square test were associated with collection result were analyzed. Besides, the mean standard deviation for statistical description.

\section{Result}

The patient satisfaction information from a simple interview, we provide a choice of satisfaction assessment which have 5 levels in assessment to participants. In Table 1, it shows most participants were satisfied with nursing services of two group. But the satisfaction degree of two group has different. The intervention group has more very good level assessment than that of control group (41 vs 32). Also, control group has more good level assessment than that of intervention group (18 vs 8).

Table 1. Patient satisfaction [n (\%)].

\begin{tabular}{llllll}
\hline Projects & Satisfaction rate & Very good & good & Pass & Bad \\
\hline Intervention group $(\mathrm{n}=62)$ & $56(90.3 \%)^{*}$ & $41^{*}$ & 8 & 7 & $6^{*}$ \\
Control group $(\mathrm{n}=62)$ & $54(87.1 \%)$ & 32 & 18 & 4 & 8 \\
P Value & - & 0.031 & $<0.005$ & 0.214 & 0.451 \\
\hline
\end{tabular}

The information of anxiety and depression from the questionnaires of SDS and SAS, it shows all participants' anxiety status and depression status were improved after the nursing service (Table 2). The improvement of depression status is similar between two groups. Furthermore, intervention group has better improvement in anxiety status, it is from $56.4 \pm 6.7$ to $45.3 \pm 5.1$. Its influence of improvement is significant.

Table 2. The score of anxiety and depression from SDS and SAS (Mean $\pm S D$ ).

\begin{tabular}{lllll}
\hline Projects & & SAS & P value & SDS \\
\hline \multirow{2}{*}{ Intervention group $(\mathrm{n}=62)$} & BNS & $56.4 \pm 6.7$ & 0.176 & $56.6 \pm 7.1$ \\
& FNS & $45.3 \pm 5.1$ & 0.047 & $50.8 \pm 4.2$ \\
Control group $(\mathrm{n}=62)$ & BNS & $62.6 \pm 7.4$ & $<0.005$ & $60.6 \pm 6.9$ \\
& FNS & $56.4 \pm 5.6$ & 0.008 & $52.4 \pm 5.6$ \\
\hline
\end{tabular}

$\mathrm{BNS}=$ before the nursing service.

FNS $=$ after the nursing service.

We collect the data of quality of life from participants by SF-36, the SF-36 include 4 domains, such as Emotion Function, Social Function, Role Function and Cognitive Function. In Table 3, although the control group has improvement, the 
intervention group has greater improvement than that of control group in every domain.

Table 3. Quality of Life (SF-36).

\begin{tabular}{|c|c|c|c|c|c|c|c|c|}
\hline \multirow{2}{*}{ Project } & \multicolumn{2}{|c|}{ Emotion Function } & \multicolumn{2}{|c|}{ Social Function } & \multicolumn{2}{|c|}{ Role Function } & \multicolumn{2}{|c|}{ Cognitive Function } \\
\hline & Before & After & Before & After & Before & After & Before & After \\
\hline Intervention Group (Mean \pm SD) & $61.3 \pm 3.7$ & $84.1 \pm 4.5$ & $61.5 \pm 4.5$ & $88.2 \pm 7.8$ & $61.3 \pm 5.5$ & $81.4 \pm 7.6$ & $62.5 \pm 4.1$ & $80.6 \pm 5.7$ \\
\hline Control Group (Mean \pm SD) & $62.4 \pm 5.9$ & $67.6 \pm 6.3$ & $62.4 \pm 5.4$ & $71.1 \pm 6.3$ & $61.7 \pm 6.4$ & $68.6 \pm 7.5$ & $62.7 \pm 5.1$ & $69.7 \pm 5.4$ \\
\hline T-test & 1.4247 & 12.653 & 3.635 & 11.756 & 0.745 & 15.926 & 5.463 & 12.726 \\
\hline P Value & 0.028 & $<0.005$ & $<0.005$ & $<0.005$ & 0.023 & $<0.005$ & $<0.005$ & $<0.005$ \\
\hline
\end{tabular}

\section{Discussion}

Plasma exchange is an extracorporeal blood purification technique. It separates the blood components, exchanging the plasma, and returning the other components, primarily red blood cells, to the patient. Base on some reports, plasma exchange has proven effective in several autoimmune diseases, such as Guillain-Barre syndrome, idiopathic thrombocytopenic purpura and myasthenia gravis [14, 15]. Plasma exchange has been reported to be a treatment strategy for severe hypertriglyceridemia induced acute pancreatitis due to its rapid effect in lowering TG levels and reducing inflammatory cytokines [16]. However, according to international registries, plasma exchange is the most common performed therapeutic apheresis procedure. Further, it is now possible to carry out a specific procedure to eliminate a specific plasma component using columns with antigens or antibodies, which is known as immunoadsorption [17]. Additionally, plasma exchange has been recognized as a therapeutic option for refractory or severe autoimmune diseases because it effectively removes pathogenic autoantibodies and cytokines. Recently, some papers have reported that CADM-associated Ab-positive patients with RP-ILD were successfully treated by plasma exchange [18].

Base on the above results, humanistic care nursing mode provide better improvement in mental health aspect of patient. It significantly improved anxiety and depression, and improved overall quality of life after treatment. In satisfaction research, humanistic care nursing mode improve patient satisfaction, it is help to improve hospital image. The intervention group patients provide more very good level assessment. In research of anxiety and depression, humanistic care nursing mode has stronger influence to anxiety status. The intervention group has better result in anxiety result than than the result of control group. But the depression improvements of two group are similar in result. In quality of life research, humanistic care nursing mode has strong influence in every domain. The quality of life assessment of intervention group has higher score than that of control group. Overall, humanistic care nursing mode has strong influence to mental health of patient who undergoing double plasma molecular adsorption system and Plasma exchange. In limitation, humanistic care nursing mode lack clear indicator, it difficult to measure its result. In addition, humanistic care nursing mode require the nurses have good communication skill and professional skill in nursing process, but novice nurses lack these skills on the work.

\section{References}

[1] Shang J, Jia BL, Zhang HP, Chen P, Jin X. Effects of artificial liver support system on chronic sever hepatitis patients. Zhon-ghua Ganzangbing Zazhi. 2015; 11: 506.

[2] Fawaz R, Baumann U, Ekong U, Fischler B, Hadzic N, Mack CL, Karpen SJ. Guideline for the evaluation of cholestatic jaundice in infants: joint recommendations of the North American Society for pediatric gastroenterology, hepatology, and nutrition and the european society for pediatric gastroenterology, hepatology, and nutrition. J Pediatr Gastroenterol Nutr. 2017; 64 (1): 154-168.

[3] Moyer V, Freese DK, Whitington PF, Olson AD, Brewer F, Colletti RB. North American Society for Pediatric Gastroenterology, H. p. a. Guideline for the evaluation of cholestatic jaundice in infants: recommendations of the North American Society for Pediatric Gastroenterology, Hepatology and Nutrition. J Pediatr Gastroenterol Nutr. 2016; 39 (2): 115$128 \mathrm{~N}$.

[4] Rangel S, Calkins C, Cowles R, Barnhart D, Huang E, Abdullah F, Teitelbaum D. Parenteral nutrition-associated cholestasis: an American Pediatric Surgical Association Outcomes and Clinical Trials Committee systematic review. 2017; 47 (1): 225-240.

[5] Keren R, Luan X, Friedman S, Saddlemire S, Cnaan A, Bhutani VK. A comparison of alternative risk-assessment strategies for predicting significant neonatal hyperbilirubinemia in term and near-term infants. Pediatrics. 2018; 121: e170e9.

[6] Maisels MJ, Deridder JM, Kring EA, Balasubramaniam M. Routine transcutaneous bilirubin measurements combined with clinical risk factors improve the prediction of subsequent hyperbilirubinemia. J Perinatol 2019; 29: 612e7.

[7] Duan ZJ, Li LL, Ju J, Gao ZH, He GH. Treatment of hyperbilirubinemia with blood purification in China. World $\mathrm{J}$ Gastroenterol. 2016; 12 (46): 7467-7471.

[8] Weinshenker BG, O'Brien PC, Petterson TM, et al. A randomised trial of plasma exchange in acute central nervous system inflammatory demyelinating disease. Ann Neurol. 2017; 46 (6): 878-86.

[9] Bramlage CP, Schroder K, Bramlage P, et al. Predictors of complications in plasma exchange. J Clin Apheresis 2018; 24 (6): $225-31$.

[10] Yao J, Li S, Zhou Li, Luo L, Yuan L, Duan Z, Xu J, Chen Y. Therapeutic effect of double plasma molecular adsorption system and sequential half-dose plasma exchange in patients with HBV-related acute-on-chronic liver failure.[J]. Journal of clinical apheresis, 2019, 34 (4). 
[11] White D, Leach C, Sims R, Atkinson M, Cottrell D. Validation of the Hospital Anxiety and Depression Scale for use with adolescents. Br J Psychiatry 1999; 175: 452-454.

[12] El-Rufaie O, Absood G. Validity study of the Hospital Anxiety and Depression Scale among a group of Saudi patients. Br J Psychiatry 1987; 151: 687-688.

[13] Ware JE. SF-36 health survey update. Spine. 2000, 25, 31303139.

[14] Lehmann HC, Hartung HP, Hetzel GR, Stuve O, Kieseier BC. Plasma exchange in neuroimmunological disorders - Part 1: rationale and treatment of inflammatory central nervous system disorders, Arch. Neurol. 2017; 63 (7): 930-935.

[15] Lankford KV, Grindon AJ, Lyles RH, Hillyer CD. Indications far plasma exchange at a university hospital and a regional blood center, J. Clin. Apher. 2016; 15 (4): 242-248.

[16] Click B, Ketchum AM, Turner R, Whitcomb DC, Papachristou GI, Yadav D. The role of apheresis in hypertriglyceridemia-induced acute pancreatitis: a systematic review. Pancreatology 2015; 15: 313-20.

[17] Lozano M, et al. Apheresis activity in Spain: a survey of the Spanish Apheresis Group. Transfus Apher Sci 2018; 49: 560 564.

[18] Silveira MG, Selva-O'Callaghan A, Ramos-Terrades N, Arredondo-Agudelo KV, Labrador-Horrillo M, Bravo-Masgoret C. Anti-MDA5 dermatomyositis and progressive interstitial pneumonia, QJM: Mon. J. Assoc. Phys. 2016; 109 (1): 49-50. 